\title{
Pacific
}

Journal of

Mathematics

\section{RESONANT SOLUTIONS AND TURNING POINTS \\ IN AN ELLIPTIC PROBLEM \\ WITH OSCILLATORY BOUNDARY CONDITIONS}

Alfonso Castro and Rosa Pardo 


\title{
RESONANT SOLUTIONS AND TURNING POINTS IN AN ELLIPTIC PROBLEM WITH OSCILLATORY BOUNDARY CONDITIONS
}

\author{
Alfonso Castro And Rosa PARdo
}

\begin{abstract}
We consider the elliptic equation $-\Delta u+u=0$ with nonlinear boundary conditions $\partial u / \partial n=\lambda u+g(\lambda, x, u)$, where the nonlinear term $g$ is oscillatory and satisfies $g(\lambda, x, s) / s \rightarrow 0$ as $|s| \rightarrow 0$. We provide sufficient conditions on $g$ for the existence of sequences of resonant solutions and turning points accumulating to zero.
\end{abstract}

\section{Introduction}

This work complements the study initiated in [Arrieta et al. 2010] and [Castro and Pardo 2011] on the positive solutions to the following boundary-value problem

$$
\begin{cases}-\Delta u+u=0 & \text { in } \Omega, \\ \frac{\partial u}{\partial n}=\lambda u+g(\lambda, x, u) & \text { on } \partial \Omega,\end{cases}
$$

where $\Omega \subset \mathbb{R}^{N}$ is a bounded and sufficiently smooth domain, $N \geq 2, \lambda$ is a real parameter, $g(\lambda, x, s)=o(s)$ as $s \rightarrow 0$ and $g$ is oscillatory. A typical example of such a $g$ is

$$
g(x, s):=s^{\alpha}\left(\sin \left|\frac{s}{\Phi_{1}(x)}\right|^{\beta}+C\right) \quad \text { with } \alpha+\beta>1, \quad \beta<0,
$$

where $\Phi_{1}$ stands for the first eigenfunction of the Steklov eigenvalue problem

$$
\begin{cases}-\Delta \Phi+\Phi=0 & \text { in } \Omega, \\ \frac{\partial \Phi}{\partial n}=\sigma \Phi & \text { on } \partial \Omega .\end{cases}
$$

Pardo is supported by the Spanish Ministerio de Ciencia e Innovación (MICINN) under Project MTM2009-07540, by UCM-BSCH, Spain, GR58/08, Grupo 920894 and also by the Programa Becas Complutense del Amo. This work was carried out during Pardo's sabbatical visit to the Department of Mathematics, Harvey Mudd College, whose hospitality she thanks.

MSC2010: primary 35B32, 35B34, 35B35, 35J25, 58J55; secondary 35J60, $35 \mathrm{~J} 65$.

Keywords: turning points, resonance, stability, instability, multiplicity, Steklov eigenvalues,

bifurcation, sublinear oscillating boundary conditions. 


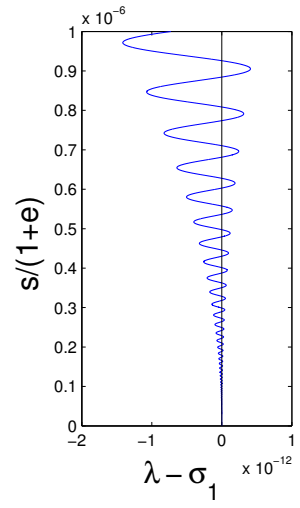

$C=0.5$

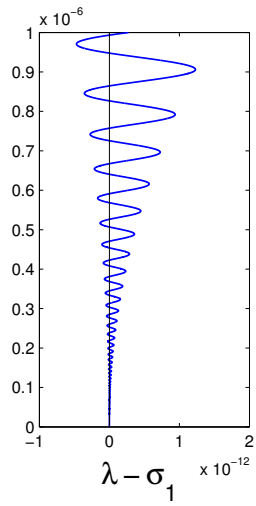

$C=-0.5$

$\alpha=3$
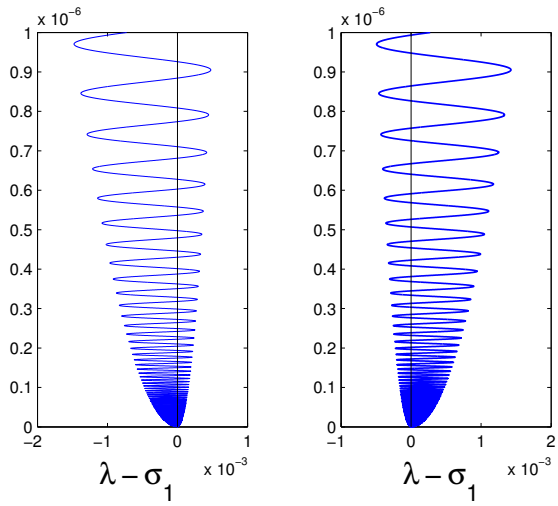

$C=0.5$

$\alpha=1.5$

Figure 1. Bifurcation diagram of subcritical and supercritical solutions, containing infinitely many turning points and infinitely many resonant solutions. In all cases, $\beta=-0.35$.

The first eigenvalue $\sigma_{1}$ is simple and, due to Hopf's lemma, we may assume its eigenfunction $\Phi_{1}$ to be strictly positive in $\bar{\Omega}$ and we take $\left\|\Phi_{1}\right\|_{L^{\infty}(\partial \Omega)}=1$.

The case $\alpha+\beta<1, \beta>0$ was treated in [Arrieta et al. 2010; Castro and Pardo 2011]. Here we focus on the case $\alpha+\beta>1, \beta<0$, inside of the complementary range. The case with $\alpha<1$ corresponds to a bifurcation from infinity phenomenon; see [Arrieta et al. 2007; 2009; 2010; Castro and Pardo 2011; Rabinowitz 1973]. In contrast, the case with $\alpha>1$ corresponds to a bifurcation from zero phenomenon; see [Arrieta et al. 2007; Crandall and Rabinowitz 1971; Rabinowitz 1971].

The oscillatory situation is in principle more complex than the monotone one, since order techniques such as sub- and supersolutions are not applicable.

One novelty in problem (1-1) is that the parameter appears explicitly in the boundary condition. With respect to this parameter, we perform an analysis of the local bifurcation diagram of nonnegative solutions to (1-1), which turns out to be different from the case $\alpha<1$ (see Figure 1 for $\alpha>1$ and Figure 2 for $\alpha<1$ ).

Throughout this paper we make the following assumptions:

(H1) $g: \mathbb{R} \times \partial \Omega \times \mathbb{R} \rightarrow \mathbb{R}$ is a Carathéodory function (i.e. $g=g(\lambda, x, s)$ is measurable in $x \in \Omega$, and continuous with respect to $(\lambda, s) \in \mathbb{R} \times \mathbb{R})$. Moreover, there exist $G_{1} \in L^{r}(\partial \Omega)$ with $r>N-1$ and continuous functions $\Lambda: \mathbb{R} \rightarrow \mathbb{R}^{+}$, and $U: \mathbb{R} \rightarrow \mathbb{R}^{+}$, satisfying

$$
\begin{cases}|g(\lambda, x, s)| \leq \Lambda(\lambda) G_{1}(x) U(s) & \text { for all }(\lambda, x, s) \in \mathbb{R} \times \partial \Omega \times \mathbb{R}, \\ \limsup _{|s| \rightarrow 0} \frac{U(s)}{|s|^{\alpha}}<+\infty & \text { for some } \alpha>1 .\end{cases}
$$



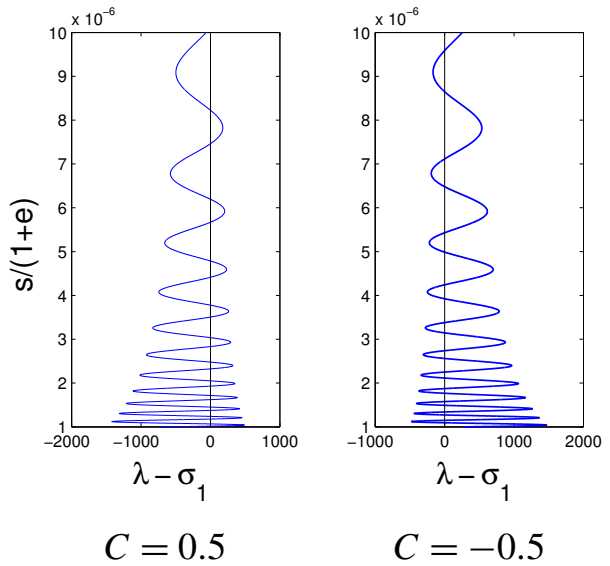

Figure 2. Bifurcation diagram in the case $\alpha=0.5, \beta=-0.35$.

(H2) The partial derivative $g_{s}(\lambda, \cdot, \cdot)$ (where $\left.g_{s}:=\partial g / \partial s\right)$ belongs to $C(\partial \Omega \times \mathbb{R})$; moreover, $g_{s}(\cdot, \cdot, 0)=0$ and there exist $F_{1} \in L^{r}(\partial \Omega)$, with $r>N-1$ and $\rho>1$ such that

$$
\frac{\left|g(\lambda, x, s)-s g_{s}(\lambda, x, s)\right|}{|s|^{\rho}} \leq F_{1}(x) \quad \text { as } \lambda \rightarrow \sigma_{1},
$$

for $x \in \partial \Omega$ and $s \leq \epsilon$ small enough.

Throughout this paper, by solutions to (1-1) we mean elements $u \in H^{1}(\Omega)$ such that

(1-4) $\int_{\Omega}(\nabla u \cdot \nabla v+u v) d x$

$$
=\lambda \int_{\partial \Omega} u v d \sigma+\int_{\partial \Omega} g(\lambda, x, u) v d \sigma \quad \text { for all } v \in H^{1}(\Omega) .
$$

As proven in [Arrieta et al. 2007, Proposition 2.3], all such solutions are in the Holder space $C^{\beta}(\bar{\Omega})$ for some $\beta>0$. Moreover, there exists a connected set of positive solutions of (1-1) known as a branch bifurcating from zero; see [Arrieta et al. 2007, Theorem 8.1]. We denote it by $\mathscr{C}^{+} \subset \mathbb{R} \times C(\bar{\Omega})$, and recall that for $\left(\lambda, u_{\lambda}\right) \in \mathscr{C}^{+}$

$$
u=s \Phi_{1}+w, \quad \text { with } \quad w=o(|s|) \quad \text { and } \quad\left|\sigma_{1}-\lambda\right|=o(1) \quad \text { as }|s| \rightarrow 0 .
$$

Definition 1.1. A solution $\left(\lambda^{*}, u^{*}\right)$ of (1-1) in the branch of solutions $\mathscr{C}^{+} \subset \mathbb{R} \times$ $C(\bar{\Omega})$ is called a turning point if there is a neighborhood $W$ of $\left(\lambda^{*}, u^{*}\right)$ in $\mathbb{R} \times C(\bar{\Omega})$ such that, either $W \cap \mathscr{C}^{+} \subset\left[\lambda^{*}, \infty\right) \times C(\bar{\Omega})$ or $W \cap \mathscr{C}^{+} \subset\left(-\infty, \lambda^{*}\right] \times C(\bar{\Omega})$.

Our goal is to give conditions on the nonlinear oscillatory term $g$ that guarantee the existence of sequences accumulating to zero of subcritical solutions (i.e., for 
values of the parameter $\lambda<\sigma_{1}$ ), supercritical solutions (i.e., for $\lambda>\sigma_{1}$ ), resonant solutions (i.e., for $\lambda=\sigma_{1}$ ), and turning points.

Our main result, Theorem 1.3 below, is exemplified by the case in which $g$ is given by (1-2). In fact we have:

Theorem 1.2. Assume that $g$ is given by (1-2) with $\beta<0$. If

$$
|C|<1 \text { and } \alpha+\beta>1,
$$

then in any neighborhood of the bifurcation point $\left(\sigma_{1}, 0\right)$ in $\mathbb{R} \times C(\bar{\Omega})$, the branch $\mathfrak{C}^{+}$of positive solutions of (1-1) contains a sequence of subcritical solutions, a sequence of supercritical solutions, a sequence of turning points, and a sequence of resonant solutions.

The proof of this follows directly from the next theorem.

Theorem 1.3. Assume the nonlinearity g satisfies hypotheses $(\mathrm{H} 1)$ and $(\mathrm{H} 2)$. Assume also that

$$
\left|\frac{g(\lambda, x, s)-g\left(\sigma_{1}, x, s\right)}{|s|^{\alpha}}\right| \rightarrow 0 \quad \text { as } \quad \lambda \rightarrow \sigma_{1}, s \rightarrow 0
$$

pointwise in $x$.

Let $G: \mathbb{R} \times C(\bar{\Omega}) \rightarrow \mathbb{R}$ be defined by

$$
G(\lambda, u):=\int_{\partial \Omega} \frac{u g(\lambda, \cdot, u)}{|u|^{1+\alpha}} \Phi_{1}^{1+\alpha} .
$$

If there exist sequences $\left\{s_{n}\right\},\left\{s_{n}^{\prime}\right\}$ converging to $0^{+}$, such that

$$
\lim _{n \rightarrow+\infty} G\left(\sigma_{1}, s_{n}^{\prime} \Phi_{1}\right)<0<\lim _{n \rightarrow+\infty} G\left(\sigma_{1}, s_{n} \Phi_{1}\right),
$$

then:

(i) For sufficiently large $n \gg 1$, if $(\lambda, u)$ is a solution of (1-1) with

$$
P(u):=\frac{\int_{\partial \Omega} u \Phi_{1}}{\int_{\partial \Omega} \Phi_{1}^{2}}=s_{n},
$$

then $(\lambda, u)$ is subcritical. Similarly, if $P(u)=s_{n}^{\prime}$ it is supercritical. Consequently, there exist two sequences of solutions of (1-1), $\left\{\left(\lambda_{n}, u_{n}\right)\right\}$ and $\left\{\left(\lambda_{n}^{\prime}, u_{n}^{\prime}\right)\right\}$ converging to $\left(\sigma_{1}, 0\right)$ as $n \rightarrow \infty$, one of them subcritical, $\lambda_{n}<\sigma_{1}$, and the other supercritical, $\lambda_{n}^{\prime}>\sigma_{1}$.

(ii) There is a sequence converging to zero of turning points $\left\{\left(\lambda_{n}^{*}, u_{n}^{*}\right)\right\}$ such that

$$
\lambda_{n}^{*} \rightarrow \sigma_{1} \quad \text { and } \quad\left\|u_{n}^{*}\right\|_{L^{\infty}(\partial \Omega)} \rightarrow 0 \quad \text { as } n \rightarrow \infty .
$$

In fact, we can always choose two subsequences of turning points, one of them subcritical, $\lambda_{2 n+1}^{*}<\sigma_{1}$, and the other supercritical, $\lambda_{2 n}^{*}>\sigma_{1}$. 
(iii) There is a sequence converging to zero of resonant solutions; i.e., there are infinitely many solutions $\left\{\left(\sigma_{1}, \widetilde{u}_{n}\right)\right\}$ of (1-1) with $\left\|\widetilde{u}_{n}\right\|_{L^{\infty}(\partial \Omega)} \rightarrow 0$.

The behavior of positive solutions to (1-1) bifurcating from $\left(\sigma_{1}, 0\right)$ described in Theorems 1.2 and 1.3 is similar to that of the solutions bifurcating from $\left(\sigma_{1}, \infty\right)$ for the sublinear problem; see [Arrieta et al. 2010] for details.

The complex nature of the nonlinearity in (1-2), makes an exhaustive analysis of the global bifurcation diagram outside the scope of this work.

In [Korman 2008] the author considers in the case $\alpha=1 \beta=1$. He assumes either $N=1$ or $\Omega$ to be a ball and the nonlinearity to be bounded by a constant small enough. He obtains what he calls an oscillatory bifurcation. We refer the reader to [García-Melián et al. 2009] for related problems with nonlinear boundary conditions.

Organization of the paper. Section 2 contains the proof of our main result, giving sufficient conditions for having subcritical, supercritical, and resonant solutions. Section 3 presents two examples; explicit resonant solutions for the oscillatory nonlinearity (1-2) and the one-dimensional case.

\section{Subcritical, supercritical and resonant solutions}

In this section we give sufficient conditions for the existence of a branch of solutions to (1-1) bifurcating from zero which is neither subcritical $\left(\lambda<\sigma_{1}\right)$, nor supercritical, $\left(\lambda<\sigma_{1}\right)$. From this, we conclude the existence of infinitely many turning points, see Definition 1.1, and an infinite number of solutions for the resonant problem, i.e. for $\lambda=\sigma_{1}$. This is achieved in Theorem 1.3

At this step, we analyze when the parameter may cross the first Steklov eigenvalue. To do that, we look at the asymptotic growth rate of the nonlinear term

$$
\underline{\boldsymbol{G}}_{0^{+}}:=\int_{\partial \Omega} \liminf _{(\lambda, s) \rightarrow\left(\sigma_{1}, 0\right)} \frac{s g(\lambda, \cdot, s)}{|s|^{1+\alpha}} \Phi_{1}^{1+\alpha}
$$

for $\alpha>1$. Changing lim inf to lim sup we define the number $\overline{\boldsymbol{G}}_{0^{+}}$. If $\underline{\boldsymbol{G}}_{0^{+}}>0$ then $\mathscr{C}^{+}$is subcritical, and if $\overline{\boldsymbol{G}}_{0^{+}}<0$ then $\mathscr{C}^{+}$is supercritical in a neighborhood of $\left(\sigma_{1}, 0\right)$ See [Arrieta et al. 2009, Theorems 3.4 and 3.5] for the bifurcation from infinity case. In this paper we consider nonlinearities for which

$$
\underline{\boldsymbol{G}}_{0^{+}}<0<\overline{\boldsymbol{G}}_{0^{+}} .
$$

We shall argue as in [Arrieta et al. 2010] for the bifurcation from infinity case. To determine whether a sequence of solutions $\left(\lambda_{n}, u_{n}\right)$ is subcritical or supercritical, 
one must check the sign of

$$
\liminf _{n \rightarrow \infty} G\left(\lambda_{n}, u_{n}\right) \text { and } \limsup _{n \rightarrow \infty} G\left(\lambda_{n}, u_{n}\right),
$$

where $G$ is defined by (1-6). This is done in Lemma 2.3.

In Proposition 2.2, it is proved that when $g$ is such that

$$
|g(\lambda, x, s)|=O\left(|s|^{\alpha}\right) \text { as }|s| \rightarrow 0 \text { for some } \alpha>1,
$$

then the solutions in $\mathscr{C}^{ \pm}$can be described as

$u_{n}=s_{n} \Phi_{1}+w_{n}, \quad$ where $\quad \int_{\partial \Omega} w_{n} \Phi_{1}=0 \quad$ and $\quad w_{n}=O\left(\left|s_{n}\right|^{\alpha}\right)$ as $n \rightarrow 0$.

We unveil the signs of the expressions in (2-2) by just looking at the signs of the expressions in (2-2) at $\lambda_{n}=\sigma_{1}$ and $u_{n}=s_{n} \Phi_{1}$ This is achieved in Lemma 2.4.

For this we first consider a family of linear Steklov problems with a variable nonhomogeneous term at the boundary $h$ depending on the parameter $\lambda$

$$
\left\{\begin{array}{lr}
-\Delta u+u=0 & \text { in } \Omega, \\
\frac{\partial u}{\partial n}=\lambda u+h(\lambda, x) & \text { on } \partial \Omega,
\end{array}\right.
$$

where $h(\lambda, \cdot) \in L^{r}(\partial \Omega), r>N-1$ and $\lambda \in\left(-\infty, \sigma_{2}\right)$.

We use the decomposition

$$
L^{r}(\partial \Omega)=\operatorname{span}\left[\Phi_{1}\right] \oplus \operatorname{span}\left[\Phi_{1}\right]^{\perp},
$$

where

$$
\operatorname{span}\left[\Phi_{1}\right]^{\perp}:=\left\{u \in L^{r}(\partial \Omega): \int_{\partial \Omega} u \Phi_{1}=0\right\} .
$$

For $h(\lambda, \cdot) \in L^{r}(\partial \Omega)$, with $r>N-1$, we write

$$
h(\lambda, \cdot)=a_{1}(\lambda) \Phi_{1}+h_{1}(\lambda, \cdot),
$$

with

$$
a_{1}(\lambda)=\frac{\int_{\partial \Omega} h(\lambda, \cdot) \Phi_{1}}{\int_{\partial \Omega} \Phi_{1}^{2}}, \quad \int_{\partial \Omega} h_{1}(\lambda, \cdot) \Phi_{1}=0 .
$$

For $\lambda \neq \sigma_{1}$ the solution $u=u(\lambda)$ of (2-3) has a unique decomposition

$$
u=\frac{a_{1}(\lambda)}{\sigma_{1}-\lambda} \Phi_{1}+w, \quad \text { where } \int_{\partial \Omega} w \Phi_{1}=0,
$$

and $w=w(\lambda) \in \operatorname{span}\left[\Phi_{1}\right]^{\perp}$ solves the problem

$$
\left\{\begin{array}{lr}
-\Delta w+w=0 & \text { in } \Omega, \\
\frac{\partial w}{\partial n}=\lambda w+h_{1}(\lambda, x) & \text { on } \partial \Omega .
\end{array}\right.
$$


Note that in (2-6) $w(\lambda) \in \operatorname{span}\left[\Phi_{1}\right]^{\perp}$ is also well defined for $\lambda=\sigma_{1}$. Moreover:

Lemma 2.1. For each compact set $K \subset\left(-\infty, \sigma_{2}\right) \subset \mathbb{R}$ there exists a constant $C=C(K)$, independent of $\lambda$, such that

$$
\|w(\lambda)\|_{L^{\infty}(\partial \Omega)} \leq C\left\|h_{1}(\lambda, \cdot)\right\|_{L^{r}(\partial \Omega)} \quad \text { for any } \lambda \in K,
$$

where $w \in \operatorname{span}\left[\Phi_{1}\right]^{\perp}$ is the solution of (2-6) and $h_{1} \in \operatorname{span}\left[\Phi_{1}\right]^{\perp}$ is defined in $(2-4)$.

Proof. See Lemma 3.1 of [Arrieta et al. 2010].

Now we turn our attention to the nonlinear problem (1-1). Recall that for solutions $(\lambda, u)$ close to the bifurcation point $\left(\sigma_{1}, 0\right)$ we have

$$
u=s \Phi_{1}+w, \quad \text { where } w \in \operatorname{span}\left[\Phi_{1}\right]^{\perp}
$$

satisfies

$$
w=o(s) \quad \text { as } s \rightarrow 0 .
$$

We define

$$
P(u):=\frac{\int_{\partial \Omega} u(\cdot) \Phi_{1}}{\int_{\partial \Omega} \Phi_{1}^{2}} .
$$

Next, we give sufficient conditions on the nonlinear term $g$ in (1-1), for $w=$ $O\left(|s|^{\alpha}\right)$ as $s \rightarrow 0$; compare (2-8). We restrict ourselves below to the branch of positive solutions; a completely analogous result holds for the branch of negative solutions. The next result is essentially Proposition 3.2 in [Arrieta et al. 2010] rewritten for $s \rightarrow 0$; we include the proof for completeness.

Proposition 2.2. Assume $g$ satisfies hypotheses $(\mathrm{H} 1)$ and $(\mathrm{H} 2)$. There exists an open set $0 \subset \mathbb{R} \times C(\bar{\Omega})$ of the form $\mathcal{O}=\left\{(\lambda, u):\left|\lambda-\sigma_{1}\right|<\delta_{0},\|u\|_{L^{\infty}(\Omega)}<s_{0}\right\}$, for some $\delta_{0}$ and $s_{0}$, satisfying these conditions:

(i) There exists a constant $C_{1}$ independent of $\lambda$ such that, if $(\lambda, u) \in \mathscr{C}^{+} \cap \mathbb{O}$ and $(\lambda, u) \neq\left(\sigma_{1}, 0\right)$ then $u=s \Phi_{1}+w$, where $s>0, w \in \operatorname{span}\left[\Phi_{1}\right]^{\perp}$ and

$$
\|w\|_{L^{\infty}(\partial \Omega)} \leq C_{1}\left\|G_{1}\right\|_{L^{r}(\partial \Omega)}|s|^{\alpha} \quad \text { as }|s| \rightarrow 0 .
$$

(ii) There exists a constant $S_{0}>0$ such that for all $|s| \leq S_{0}$ there exists $(\lambda, u)$ in $\mathscr{C}^{+} \cap \mathcal{O}$ satisfying $u=s \Phi_{1}+w$, with $w \in \operatorname{span}\left[\Phi_{1}\right]^{\perp}$.

(iii) Moreover, for any $(\lambda, u) \in \mathscr{C}^{+} \cap \mathcal{O}, u=s \Phi_{1}+w$, with $w \in \operatorname{span}\left[\Phi_{1}\right]^{\perp}$,

$$
\left|\sigma_{1}-\lambda\right| \leq C_{2}|s|^{\alpha-1} \quad \text { as }|s| \rightarrow 0,
$$


with $C_{2}$ independent of $\lambda$; in fact,

$$
C_{2}=\frac{2\left\|G_{1}\right\|_{L^{1}(\partial \Omega)}}{\int_{\partial \Omega} \Phi_{1}^{2}}
$$

Proof. From (2-7) and (2-8), we have $\Phi_{1}+w / s \rightarrow \Phi_{1}$ as $s \rightarrow 0$ in $L^{\infty}(\partial \Omega)$. Together with (H1) and Lemma 2.1, this implies that $\|w\|_{L^{\infty}(\partial \Omega)} \leq C|s|^{\alpha}$ as $s \rightarrow 0$. This proves part (i).

To prove part (ii) note that $\mathscr{C}^{+} \cap \mathcal{O}$ is connected. Hence, using the decomposition in (2-7), we have $u=s \Phi_{1}+w$ with $w \in \operatorname{span}\left[\Phi_{1}\right]^{\perp}$. Since the projection $P$ is continuous, by (2-9), the set

$$
\left\{s \in \mathbb{R}:(1-1) \text { has a solution of the form } u=s \Phi_{1}+w \text { and } w \in\left[\operatorname{span}\left[\Phi_{1}\right]^{\perp}\right]\right\}
$$

contains an interval in $\mathbb{R}$ containing zero.

To prove part (iii) we observe that if $(\lambda, u)$ is a solution of (1-1), $u={ }_{s} \Phi_{1}+w$, with $w \in \operatorname{span}\left[\Phi_{1}\right]^{\perp}$, multiplying the equation by the first Steklov eigenfunction $\Phi_{1}>0$ and integrating by parts we obtain,

$$
\left(\sigma_{1}-\lambda\right) s \int_{\partial \Omega} \Phi_{1}^{2}=\int_{\partial \Omega} g\left(\lambda, x, s \Phi_{1}+w\right) \Phi_{1} .
$$

Taking into account that

$$
\frac{\left|g\left(\lambda, x, s \Phi_{1}+w\right)\right|}{|s|}=\frac{\left|g\left(\lambda, x, s \Phi_{1}+w\right)\right|}{\left|s \Phi_{1}+w\right|}\left|\Phi_{1}+\frac{w}{s}\right| \rightarrow 0 \quad \text { as } s \rightarrow 0
$$

we get $\lambda \rightarrow \sigma_{1}$ as $s \rightarrow 0$.

Moreover, from (H1), we obtain that

$$
\begin{aligned}
\left|g\left(\lambda, x, s \Phi_{1}+w\right)\right| & =|s|^{\alpha} \frac{\left|g\left(\lambda, x, s \Phi_{1}+w\right)\right|}{\left|s \Phi_{1}+w\right|^{\alpha}}\left|\Phi_{1}+\frac{w}{s}\right|^{\alpha} \\
& \leq C|s|^{\alpha} G_{1}(x)\left|\Phi_{1}+\frac{w}{s}\right|^{\alpha}
\end{aligned}
$$

and therefore

$$
\left|\sigma_{1}-\lambda\right| \leq C \frac{|s|^{\alpha-1}}{\int_{\partial \Omega} \Phi_{1}^{2}} \int_{\partial \Omega} G_{1}(x)\left|\Phi_{1}+\frac{w}{s}\right|^{\alpha} \Phi_{1} \leq C\left\|G_{1}\right\|_{L^{r}(\partial \Omega)}|s|^{\alpha-1},
$$

which ends the proof.

Our next result is essentially Lemma 3.1 in [Arrieta et al. 2009] rewritten for $s \rightarrow 0$. It allows us to estimate $\sigma_{1}-\lambda_{n}$ as $\lambda_{n}$ converges $\sigma_{1}$.

Lemma 2.3. Assume the nonlinearity $g$ satisfies hypotheses $(\mathrm{H} 1)$ and $(\mathrm{H} 2)$. Let $\left(\lambda_{n}, u_{n}\right)$ be a sequence of solutions of (1-1) with $\lambda_{n} \rightarrow \sigma_{1}$ and $\left\|u_{n}\right\|_{L^{\infty}(\partial \Omega)} \rightarrow 0$. 
If $u_{n}>0$ then

$$
\begin{aligned}
\frac{\underline{\boldsymbol{G}}_{0^{+}}}{\int_{\partial \Omega} \Phi_{1}^{2}} & \leq \frac{1}{\int_{\partial \Omega} \Phi_{1}^{2}} \liminf _{n \rightarrow \infty} G\left(\lambda_{n}, u_{n}\right) \\
& \leq \liminf _{n \rightarrow \infty} \frac{\sigma_{1}-\lambda_{n}}{\left\|u_{n}\right\|_{L^{\infty}(\partial \Omega)}^{\alpha-1}} \leq \limsup _{n \rightarrow \infty} \frac{\sigma_{1}-\lambda_{n}}{\left\|u_{n}\right\|_{L^{\infty}(\partial \Omega)}^{\alpha-1}} \\
& \leq \frac{1}{\int_{\partial \Omega} \Phi_{1}^{2}} \limsup _{n \rightarrow \infty} G\left(\lambda_{n}, u_{n}\right) \leq \frac{\overline{\boldsymbol{G}}_{0^{+}}}{\int_{\partial \Omega} \Phi_{1}^{2}} .
\end{aligned}
$$

A similar statement is obtained for the case $u_{n}<0$, just replacing $\underline{\boldsymbol{G}}_{0^{+}}$by $\underline{\boldsymbol{G}}_{0^{-}}$ and $\overline{\boldsymbol{G}}_{0^{+}}$by $\overline{\boldsymbol{G}}_{0^{-}}$.

Proof. We show that $u_{n}>0$; the other case has a similar proof. Consider a family of solutions $u_{n}$ of (1-1) for $\lambda=\lambda_{n}$ with $\lambda_{n} \rightarrow \sigma_{1}$ and $0<u_{n} \rightarrow 0$. Multiplying (1-1) by $\Phi_{1}$ and integrating by parts, we get

$$
\left(\sigma_{1}-\lambda_{n}\right) \int_{\partial \Omega} u_{n} \Phi_{1}=\int_{\partial \Omega} g\left(\lambda_{n}, x, u_{n}\right) \Phi_{1} .
$$

But

$$
\int_{\partial \Omega} g\left(\lambda_{n}, x, u_{n}\right) \Phi_{1}=\left\|u_{n}\right\|_{L^{\infty}(\partial \Omega)}^{\alpha} \int_{\partial \Omega} \frac{g\left(\lambda_{n}, x, u_{n}\right)}{u_{n}^{\alpha}}\left(\frac{u_{n}}{\left\|u_{n}\right\|_{L^{\infty}(\partial \Omega)}}\right)^{\alpha} \Phi_{1} .
$$

Taking into account the definition of $G(\lambda, u)$ in (1-6), we can write

$$
\begin{aligned}
\int_{\partial \Omega} \frac{g\left(\lambda_{n}, x, u_{n}\right)}{u_{n}^{\alpha}}( & \left.\frac{u_{n}}{\left\|u_{n}\right\|_{L^{\infty}(\partial \Omega)}}\right)^{\alpha} \Phi_{1} \\
& =\int_{\partial \Omega} \frac{g\left(\lambda_{n}, x, u_{n}\right)}{u_{n}^{\alpha}}\left[\left(\frac{u_{n}}{\left\|u_{n}\right\|_{L^{\infty}(\partial \Omega)}}\right)^{\alpha}-\Phi_{1}^{\alpha}\right] \Phi_{1}+G\left(\lambda_{n}, u_{n}\right) .
\end{aligned}
$$

Moreover,

$$
\int_{\partial \Omega} \frac{g\left(\lambda_{n}, x, u_{n}\right)}{u_{n}^{\alpha}}\left[\left(\frac{u_{n}}{\left\|u_{n}\right\|_{L^{\infty}(\partial \Omega)}}\right)^{\alpha}-\Phi_{1}^{\alpha}\right] \Phi_{1} \rightarrow 0 \quad \text { as } n \rightarrow \infty,
$$

because $u_{n} /\left\|u_{n}\right\|_{L^{\infty}(\partial \Omega)} \rightarrow \Phi_{1}$ uniformly in $\partial \Omega$.

But, firstly from the above, secondly from Fatou's lemma, and thirdly from definition of $\underline{\boldsymbol{G}}_{0^{+}}$,

$$
\begin{aligned}
\liminf _{n \rightarrow \infty} & \int_{\partial \Omega} \frac{g\left(\lambda_{n}, x, u_{n}\right)}{u_{n}^{\alpha}}\left(\frac{u_{n}}{\left\|u_{n}\right\|_{L^{\infty}(\partial \Omega)}}\right)^{\alpha} \Phi_{1} \\
& \geq \liminf _{n \rightarrow \infty} G\left(\lambda_{n}, u_{n}\right) \geq \int_{\partial \Omega} \liminf _{n \rightarrow \infty} \frac{g\left(\lambda_{n}, x, u_{n}\right)}{u_{n}^{\alpha}} \Phi_{1}^{1+\alpha} \\
& \geq \underline{G}_{0^{+}} .
\end{aligned}
$$


Dividing both sides of (2-11) by $\left\|u_{n}\right\|_{L^{\infty}(\partial \Omega)}^{\alpha}$ and passing to the limit we obtain the first two inequalities in the chain (2-10). The third inequality in the chain is trivial and the last two are obtained in a similar manner.

Let $\left\{s_{n}\right\}$ and $\left\{s_{n}^{\prime}\right\}$ satisfy

$$
-\infty<\lim _{n \rightarrow+\infty} G\left(\sigma_{1}, s_{n}^{\prime} \Phi_{1}\right)<0<\lim _{n \rightarrow+\infty} G\left(\sigma_{1}, s_{n} \Phi_{1}\right)<\infty .
$$

In order to prove Theorem 1.3, we show that the signs in (2-2) can be deduced from those of (2-13). This is stated in the following result, which is a slight variation of [Arrieta et al. 2010, Lemma 3.3].

Lemma 2.4. Assume that $g$ satisfies hypotheses (H1), (H2), and (1-5).

If $\left(\lambda_{n}, s_{n}\right) \rightarrow\left(\sigma_{1}, 0\right)$ and there exists a constant $C$ such that $\left\|w_{n}\right\|_{L^{\infty}(\partial \Omega)} \leq C\left|s_{n}\right|^{\alpha}$ for all $n \rightarrow 0$, then

$$
\liminf _{n \rightarrow+\infty} G\left(\lambda_{n}, s_{n} \Phi_{1}+w_{n}\right) \geq \liminf _{n \rightarrow+\infty} G\left(\sigma_{1}, s_{n} \Phi_{1}\right),
$$

where $G$ is given by (1-6). Similarly,

$$
\limsup _{n \rightarrow+\infty} G\left(\lambda_{n}, s_{n} \Phi_{1}+w_{n}\right) \leq \limsup _{n \rightarrow+\infty} G\left(\sigma_{1}, s_{n} \Phi_{1}\right) .
$$

Proof. Throughout this proof, $C$ denotes several constants depending only on $(\Omega, g)$. Given $\varepsilon>0$, assume that $\left|\left(\lambda_{n}, s_{n}\right)-\left(\sigma_{1}, 0\right)\right|<\varepsilon$.

By the mean value theorem we have

$$
\begin{aligned}
g\left(\lambda_{n}, x, s_{n} \Phi_{1}+w_{n}\right) & -g\left(\lambda_{n}, x, s_{n} \Phi_{1}\right) \\
& =w_{n} \int_{0}^{1} g_{s}\left(\lambda_{n}, \cdot, s_{n} \Phi_{1}+\tau w_{n}\right) d \tau \\
\leq & \left\|w_{n}\right\|_{L^{\infty}(\partial \Omega)} \sup _{\tau \in[0,1]}\left\|g_{s}\left(\lambda_{n}, \cdot, s_{n} \Phi_{1}+\tau w_{n}\right)\right\|_{L^{\infty}(\partial \Omega)} .
\end{aligned}
$$

Therefore

$$
\begin{aligned}
\int_{\partial \Omega} \mid g\left(\lambda_{n}, x,\right. & \left.s_{n} \Phi_{1}+w_{n}\right)-g\left(\lambda_{n}, x, s_{n} \Phi_{1}\right) \mid \Phi_{1} d x \\
& \leq\left\|w_{n}\right\|_{L^{\infty}(\partial \Omega)} \int_{\partial \Omega} \sup _{\tau \in[0,1]}\left\|g_{s}\left(\lambda_{n}, \cdot, s_{n} \Phi_{1}+\tau w_{n}\right)\right\|_{L^{\infty}(\partial \Omega)} \\
& \leq|\partial \Omega|\left\|w_{n}\right\|_{L^{\infty}(\partial \Omega)} \sup _{\tau \in[0,1]}\left\|g_{s}\left(\lambda_{n}, \cdot, s_{n} \Phi_{1}+\tau w_{n}\right)\right\|_{L^{\infty}(\partial \Omega)} .
\end{aligned}
$$

By hypotheses (H1) and (H2), for all $x \in \partial \Omega$,

$$
\begin{aligned}
& \frac{\left|g_{s}\left(\lambda_{n}, x, s\right)\right|}{|s|^{\gamma-1}} \\
& \quad \leq|s|^{\rho-\gamma} F_{1}(x)+C|s|^{\alpha-\gamma} G_{1}(x) \max \left\{\Lambda\left(\lambda_{n}\right), n \geq 1\right\}=: D_{1}(x),
\end{aligned}
$$


for $n$ large, and $\gamma=\min \{\rho, \alpha\}>1$ Hence, $D_{1} \in L^{r}(\partial \Omega)$ with $r>N-1$ and

$$
\sup _{|s| \leq 1 / n}\left|g_{s}\left(\lambda_{n}, x, s\right)\right| \leq D_{1}(x)\left(\frac{1}{n}\right)^{\gamma-1}, \quad \text { with } \gamma>1 .
$$

Since $\left\|w_{n}\right\|_{L^{\infty}(\partial \Omega)}=O\left(\left|s_{n}\right|^{\alpha}\right)$, we obtain from (2-15) and (2-17)

$$
\begin{aligned}
\int_{\partial \Omega} \frac{\mid g\left(\lambda_{n}, \cdot, s_{n} \Phi_{1}+w_{n}\right)-g}{} & \left.\lambda_{n}, \cdot, s_{n} \Phi_{1}\right) \mid \\
\left|s_{n}\right|^{\alpha} & \leq C \sup _{\tau \in[0,1]}\left\|g_{s}\left(\lambda_{n}, \cdot, s_{n} \Phi_{1}+\tau w_{n}\right)\right\|_{L^{\infty}(\partial \Omega)} \\
& \leq C \sup _{|s| \leq 1 / n}\left\|g_{s}\left(\lambda_{n}, \cdot, s\right)\right\|_{L^{\infty}(\partial \Omega)},
\end{aligned}
$$

which tends to 0 as $n \rightarrow \infty$.

Therefore

$$
\begin{aligned}
& \liminf _{n \rightarrow+\infty} \int_{\partial \Omega} \frac{s_{n} g\left(\lambda_{n}, \cdot, s_{n} \Phi_{1}+w_{n}\right)}{\left|s_{n}\right|^{1+\alpha}} \Phi_{1} \\
& \quad \geq \lim _{n \rightarrow \infty} \int_{\partial \Omega} \frac{s_{n} g\left(\lambda_{n}, \cdot, s_{n} \Phi_{1}+w_{n}\right)-s_{n} g\left(\lambda_{n}, \cdot, s_{n} \Phi_{1}\right)}{\left|s_{n}\right|^{1+\alpha}} \Phi_{1}+\liminf _{n \rightarrow+\infty} \int_{\partial \Omega} \frac{s_{n} g\left(\lambda_{n}, \cdot, s_{n} \Phi_{1}\right)}{\left|s_{n}\right|^{1+\alpha}} \Phi_{1} \\
& \quad=\liminf _{n \rightarrow+\infty} \int_{\partial \Omega} \frac{s_{n} g\left(\lambda_{n}, \cdot, s_{n} \Phi_{1}\right)}{\left|s_{n}\right|^{1+\alpha}} \Phi_{1} \\
& \quad=\liminf _{n \rightarrow+\infty} \int_{\partial \Omega} \frac{s_{n} g\left(\sigma_{1}, \cdot, s_{n} \Phi_{1}\right)}{\left|s_{n}\right|^{1+\alpha}} \Phi_{1},
\end{aligned}
$$

where we have used (2-18) and (1-5) respectively.

Now note that, multiplying and dividing by $\left|\Phi_{1}+w_{n} / s_{n}\right|^{\alpha}$ the integrand of the left hand side above can be written as

$$
\frac{s_{n} g\left(\lambda_{n}, \cdot, s_{n} \Phi_{1}+w_{n}\right)}{\left|s_{n}\right|^{1+\alpha}} \Phi_{1}=\frac{\left(s_{n} \Phi_{1}+w_{n}\right) g\left(\lambda_{n}, \cdot, s_{n} \Phi_{1}+w_{n}\right)}{\left|s_{n} \Phi_{1}+w_{n}\right|^{1+\alpha}}\left|\Phi_{1}+\frac{w_{n}}{s_{n}}\right|^{\alpha} \Phi_{1} .
$$

Then, (H2) and the fact that $\Phi_{1}+w_{n} / s_{n} \rightarrow \Phi_{1}$ in $L^{\infty}(\partial \Omega)$ concludes the proof.

Now we prove the first main result in this paper. Roughly speaking, it states that if there are a sequence of subcritical solutions and another of supercritical solutions, since the solution set is connected, there are infinitely many turning points and infinitely many resonant solutions. We prove the result for the positive branch. The same conclusions can be attained for the connected branch of negative solutions bifurcating from zero. 
Proof of Theorem 1.3. From Proposition 2.2(ii), consider any two sequences of solutions of (1-1), such that $\left(\lambda_{n}, u_{n}\right) \rightarrow\left(\sigma_{1}, 0\right)$ and $\left(\lambda_{n}^{\prime}, u_{n}^{\prime}\right) \rightarrow\left(\sigma_{1}, 0\right)$ in $\mathscr{C}^{+}$with

$$
P\left(u_{n}\right)=\frac{\int_{\partial \Omega} u_{n} \Phi_{1}}{\int_{\partial \Omega} \Phi_{1}^{2}}=s_{n} \quad \text { and } \quad P\left(u_{n}^{\prime}\right)=\frac{\int_{\partial \Omega} u_{n}^{\prime} \Phi_{1}}{\int_{\partial \Omega} \Phi_{1}^{2}}=s_{n}^{\prime} .
$$

Writing $u_{n}=s_{n} \Phi_{1}+w_{n}$, with $w_{n} \in \operatorname{span}\left[\Phi_{1}\right]^{\perp}$, from Proposition 2.2(i), we have $\left\|w_{n}\right\|_{L^{\infty}(\partial \Omega)}=O\left(\left|s_{n}\right|^{\alpha}\right)$. From Lemmata 2.3, and 2.4, hypotheses (1-5) and (1-7) we get that

$$
\begin{aligned}
\liminf _{n \rightarrow \infty} \frac{\sigma_{1}-\lambda_{n}}{\left\|u_{n}\right\|_{L^{\infty}(\partial \Omega)}^{\alpha-1}} & \geq \frac{1}{\int_{\partial \Omega} \Phi_{1}^{2}} \liminf _{n \rightarrow \infty} \int_{\partial \Omega} \frac{\left(s_{n} \Phi_{1}+w_{n}\right) g\left(\lambda_{n}, \cdot, s_{n} \Phi_{1}+w_{n}\right)}{\left|s_{n} \Phi_{1}+w_{n}\right|^{1+\alpha}} \Phi_{1}^{1+\alpha} \\
& \geq \frac{1}{\int_{\partial \Omega} \Phi_{1}^{2}} \liminf _{n \rightarrow+\infty} \int_{\partial \Omega} \frac{s_{n} g\left(\lambda_{n}, \cdot, s_{n} \Phi_{1}\right)}{\left|s_{n}\right|^{1+\alpha}} \Phi_{1} \\
& =\frac{1}{\int_{\partial \Omega} \Phi_{1}^{2}} \liminf _{n \rightarrow+\infty} \int_{\partial \Omega} \frac{s_{n} g\left(\sigma_{1}, \cdot, s_{n} \Phi_{1}\right)}{\left|s_{n}\right|^{1+\alpha}} \Phi_{1}>0,
\end{aligned}
$$

and therefore $\lambda_{n}<\sigma_{1}$.

Analogously, for $\left(\lambda_{n}^{\prime}, u_{n}^{\prime}\right)$ we get $\lambda_{n}^{\prime}>\sigma_{1}$. Hence (i) is proved.

To prove (ii), assume, by choosing subsequences if necessary, that $s_{n}>s_{n}^{\prime}>s_{n+1}$ for all $n \geq 0$ and that $0<s_{n}, s_{n}^{\prime} \leq S_{0}$ where $S_{0}$ is the one from Proposition 2.2(ii). In particular, by parts (i) and (ii) of Proposition 2.2, if $(\lambda, u) \in \mathscr{C}^{+}$and $P(u)=s<S_{0}$ then $\|u\|_{L^{\infty}(\partial \Omega)} \leq\left(1+C_{1}\left\|G_{1}\right\|_{L^{r}(\partial \Omega)}\left|S_{0}\right|^{\alpha-1}\right) s$. Taking $S_{0}$ small enough we may assume that $\|u\|_{L^{\infty}(\partial \Omega)} \leq 2 s$.

Let

$$
K_{n}=\left\{(\lambda, u) \in \mathscr{C}^{+}: P(u)=s \text { and } s_{n} \geq s \geq s_{n+1}\right\} .
$$

Let us see that, for each $n \in \mathbb{N}, K_{n}$ is a compact subset of $\mathbb{R} \times C(\bar{\Omega})$. Let $\left\{\left(\mu_{k}, v_{k}\right)\right\}$ be a sequence in $K_{n}$. Without loss of generality we may assume that $\left\{\mu_{k}\right\}$ converges to $\mu^{*}$. Since $v_{k}=t_{k} \Phi_{1}+w_{k}$ with $w_{k}=O\left(\left|t_{k}\right|^{\alpha}\right)$ and $s_{n} \geq t_{k}=$ : $P\left(v_{k}\right) \geq s_{n+1}$, for all $k$, we have $\left\|v_{k}\right\|_{C(\partial \Omega)} \leq t_{k}+\left\|w_{k}\right\|_{C(\partial \Omega)} \leq C$ with $C$ independent of $k$. This together with Proposition 2.3 of [Arrieta et al. 2007] yields

$$
\left\|v_{k}\right\|_{C(\bar{\Omega})} \leq C_{1}\left(1+\left\|v_{k}\right\|_{C(\partial \Omega)}\right) \leq C,
$$

where, again, $C$ is independent of $k$. Since the embedding $C^{\gamma}(\bar{\Omega}) \rightarrow C^{\gamma^{\prime}}(\bar{\Omega})$ is compact for $0<\gamma^{\prime}<\gamma$ we may further assume that the sequence $\left\{v_{k}\right\}$ converges to some $u^{*} \in C^{\gamma^{\prime}}(\bar{\Omega})$. This, hypothesis (H1) and the dominated convergence theorem imply that $\left\{g\left(\mu_{k}, \cdot, v_{k}\right)\right\}$ converges to $g\left(\mu^{*}, \cdot, u^{*}\right)$ in $L^{r}(\partial \Omega)$. Therefore, since

$$
\begin{cases}-\Delta v_{k}+v_{k}=0 & \text { in } \Omega \\ \frac{\partial v_{k}}{\partial n}=\mu_{k} v_{k}+g\left(\mu_{k}, x, v_{k}\right) & \text { on } \partial \Omega,\end{cases}
$$


passing to the limit in the weak sense we have

$$
\begin{cases}-\Delta u^{*}+u^{*}=0 & \text { in } \Omega, \\ \frac{\partial u^{*}}{\partial n}=\mu^{*} u^{*}+g\left(\lambda^{*}, x, u^{*}\right) & \text { on } \partial \Omega .\end{cases}
$$

By the continuity of the projection operator we also have $s_{n} \geq s^{*}=P(u *)=$ $\lim _{k \rightarrow \infty} P\left(v_{k}\right) \geq s_{n+1}$. Hence $\left(\mu^{*}, u^{*}\right) \in K_{n}$, which proves that $K_{n}$ is compact.

Since $s_{n}>s_{n}^{\prime}>s_{n+1}$ there exists $(\lambda, u) \in K_{n}$ with $\lambda>\sigma_{1}$. Hence, if we define

$$
\lambda_{n}^{*}=\sup \left\{\lambda:(\lambda, u) \in K_{n}\right\},
$$

then $\lambda_{n}^{*} \geq \lambda_{n}^{\prime}>\sigma_{1}$ see part (i). From the compactness of $K_{n}$ there exists $u_{n}^{*}$ such that $\left(\lambda_{n}^{*}, u_{n}^{*}\right) \in K_{n}$. From the definition of $\lambda_{n}^{*}$ if $(\lambda, u)$ is a solution of (1-1) with $s_{n}>P\left(u_{n}\right)>s_{n+1}$, then $\lambda \leq \lambda_{n}^{*}$ which proves that $\left(\lambda_{n}^{*}, u_{n}^{*}\right)$ is a (supercritical) turning point.

With a completely symmetric argument, using the sets

$$
K_{n}^{\prime}=\left\{(\lambda, u) \in \mathscr{C}^{+}: P(u)=s \text { and } s_{n}^{\prime} \geq s \geq s_{n+1}^{\prime}\right\}
$$

and defining $\lambda_{n}^{\prime *}=\inf \left\{\lambda:(\lambda, u) \in K_{n}^{\prime}\right\}$, we show the existence of $u_{*}$ such that $\left(\lambda_{n}^{\prime *}, u_{n}^{\prime *}\right) \in K_{n}^{\prime}$ is a (subcritical) turning point.

In order to prove the existence of resonant solutions, we now show that there exists $n_{0} \in \mathbb{N}$ such that for each $n \geq n_{0}$ both sets $K_{n}$ and $K_{n}^{\prime}$ contain resonant solutions, that is, solutions of the form $\left(\sigma_{1}, u\right)$.

We use a reductio ad absurdum argument for the sets $K_{n}$. If this is not the case, then there will exist a sequence of integers numbers $n_{j} \rightarrow+\infty$ such that $K_{n_{j}}$ does not contain any resonant solution. This implies that the compact sets $K_{n_{j}}^{+}=\left\{(\lambda, u) \in K_{n_{j}}: \lambda \geq \sigma_{1}\right\}$ can be written as

$$
K_{n_{j}}^{+}=\mathscr{C}^{+} \cap\left\{(\lambda, u) \in \mathbb{R} \times C(\partial \Omega): \lambda>\sigma_{1}, s_{n_{j}}>P(u)>s_{n_{j}+1}\right\} ;
$$

therefore $K_{n_{j}}^{+}$contains at least a connected component of $\mathscr{C}^{+}$. Moreover it is nonempty since we know that there exists at least one solution $(\lambda, u)$ with $P(u)=$ $s_{n_{j}}^{\prime} \in\left(s_{n_{j}}, s_{n_{j}+1}\right)$ and therefore $\lambda>\sigma_{1}$. The fact that we can construct a sequence of connected components of $\mathscr{C}^{+}$contradicts the fact that $\mathscr{C}^{+}$is a connected near $\left(\sigma_{1}, 0\right) \in \mathbb{R} \times C(\bar{\Omega})$.

A completely symmetric argument can be applied to the sets $K_{n}^{\prime}$.

\section{Two examples}

3.1. Resonant solutions for the oscillatory nonlinearity (1-2). In [Arrieta et al. 2007, Theorem 8.1] it is proved that if $\alpha>1$, for any $\beta \in \mathbb{R}$, and $C \in \mathbb{R}$ there is an unbounded branch of positive solutions. Assume from now that $\beta<0$. 
Taking $|C| \leq 1$ it is not difficult to see that

$$
u_{k}(x):=[\operatorname{asin}(-C)+k \pi]^{1 / \beta} \Phi_{1}(x), \quad k \geq 0,
$$

defines a sequence of resonant solutions to (1-1) such that $u_{k}(x) \rightarrow 0$ as $k \rightarrow \infty$.

3.2. A one-dimensional example. Now we consider the one-dimensional version of (1-1), where most computations can be made explicit.

Let $\left\{\sigma_{i}\right\}$ denote the sequence of Steklov eigenvalues of the problem (1-3). For $N>1$ the Steklov eigenvalues form an increasing sequence of real numbers, $\left\{\sigma_{i}\right\}_{i=1}^{\infty}$ while for $N=1$ there are only two Steklov eigenvalues as we made explicit below.

Observe that Equation (1-1) in the one-dimensional domain $\Omega=(0,1)$ reads

$$
\left\{\begin{aligned}
-u_{x x}+u=0 \quad \text { in }(0,1), \\
-u_{x}(0)=\lambda u+g(\lambda, 0, u(0)), \\
u_{x}(1)=\lambda u+g(\lambda, 1, u(1)) .
\end{aligned}\right.
$$

The general solution of the differential equation is $u(x)=a e^{x}+b e^{-x}$ and therefore the nonlinear boundary conditions provide two nonlinear equations in terms of two constants $a$ and $b$. The function $u=a e^{x}+b e^{-x}$ is a solution if $(\lambda, a, b)$ satisfy

$$
\left(\begin{array}{cc}
-(1+\lambda) & (1-\lambda) \\
(1-\lambda) e & -(1+\lambda) e^{-1}
\end{array}\right)\left(\begin{array}{l}
a \\
b
\end{array}\right)=\left(\begin{array}{c}
g(\lambda, 0, a+b) \\
g\left(\lambda, 1, a e+b e^{-1}\right)
\end{array}\right) .
$$

In this case we only have two Steklov eigenvalues,

$$
\sigma_{1}=\frac{e-1}{e+1}<\sigma_{2}=\frac{1}{\sigma_{1}}=\frac{e+1}{e-1} .
$$

Restricting the analysis to symmetric solutions $u_{s}(x)=s\left(e^{x}+e^{1-x}\right)$, with $s \in \mathbb{R}$, and choosing $g(\lambda, x, s)=g(s)$, it is easy to prove that $u_{s}(x)$ is a solution if and only if $\lambda$ satisfies

$$
\lambda(s)=\sigma_{1}-\frac{g(s(e+1))}{s(e+1)}, \quad s>0 .
$$

Therefore, whenever $g(u)=o(u)$ at zero, there is a branch of solutions $\left(\lambda(s), u_{s}\right)$ converging to $\left(\sigma_{1}, 0\right)$ as $s \rightarrow 0$.

Now fix $g(s)=s^{\alpha} \sin \left(s^{\beta}\right)$ for an arbitrary $\alpha>1, \beta<0$. From the definition in (2-1) we can write

$$
\begin{aligned}
& \underline{\boldsymbol{G}}_{0^{+}}:=\int_{\partial \Omega} \liminf _{s \rightarrow 0^{+}} \frac{s g(s)}{|s|^{1+\alpha}} \Phi^{1+\alpha}=\int_{\partial \Omega} \liminf _{s \rightarrow 0^{+}} \sin \left(s^{\beta}\right) \Phi^{1+\alpha}=-\int_{\partial \Omega} \Phi^{1+\alpha}<0, \\
& \overline{\boldsymbol{G}}_{0^{+}}:=\int_{\partial \Omega} \limsup _{s \rightarrow 0^{+}} \frac{s g(s)}{|s|^{1+\alpha}} \Phi^{1+\alpha}=\int_{\partial \Omega} \limsup _{s \rightarrow 0^{+}} \sin \left(s^{\beta}\right) \Phi^{1+\alpha}=\int_{\partial \Omega} \Phi^{1+\alpha}>0,
\end{aligned}
$$


and then $\underline{\boldsymbol{G}}_{0^{+}}<0<\overline{\boldsymbol{G}}_{0^{+}}$.

Moreover, by looking in (3-1) at the values of $s \in \mathbb{R}$ such that $\lambda(s)=\sigma_{1}$ it is easy to check that $\left(\sigma_{1}, u_{k}\right)$ is a solution for any $k \in \mathbb{Z}$, where

$$
u_{k}(x):=\frac{(k \pi)^{1 / \beta}}{e+1}\left(e^{x}+e^{1-x}\right)
$$

that is, there is a sequence of solutions of the resonant problem converging to zero, as shown in Figure 3.

Moreover, computing in (3-1) the local maxima and minima of $\lambda(s)$ it is not difficult to check that $\left(\lambda_{k}^{*}, u_{k}^{*}\right)$ is a sequence of turning points converging to zero, where

$$
\lambda_{k}^{*}:=\sigma_{1}-t_{k}^{(\alpha-1) / \beta} \sin \left(t_{k}\right), \quad u_{k}^{*}(x):=t_{k}^{1 / \beta}\left(e^{x}+e^{1-x}\right)
$$

and where $t_{k}$ is such that

$$
\tan \left(t_{k}\right)=-\frac{\beta}{\alpha-1} t_{k}, \quad t_{k} \in[-\pi / 2+k \pi, \pi / 2+k \pi]
$$

with $t_{k} \rightarrow \infty$ and $t_{k}^{1 / \beta} \rightarrow 0$ as $k \rightarrow \infty$ thanks to $\beta<0$.

Let us observe that the bifurcation from zero phenomena occurs whenever $\alpha>1$ for any $\beta$ and that whenever $\alpha+\beta<1$ the number of oscillations grows up faster than the number of oscillations of multiples of the eigenfunction and cannot be controlled; compare the two parts of Figure 3.
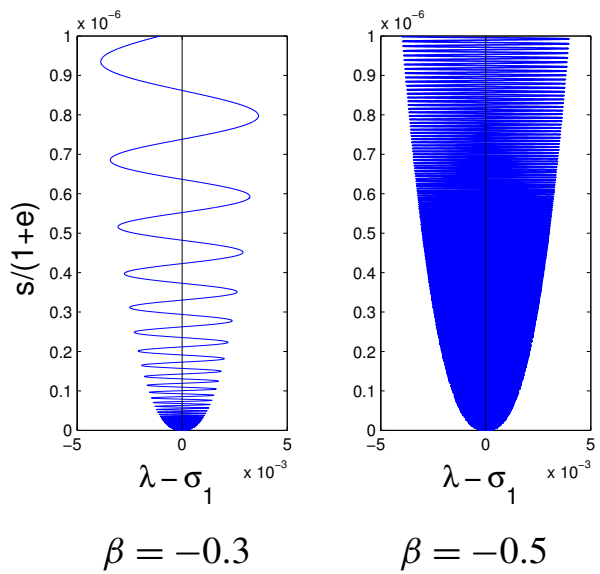

Figure 3. Bifurcation diagram in the case $\alpha=1.4$, for two values of $\beta$. 


\title{
References
}

[Arrieta et al. 2007] J. M. Arrieta, R. Pardo, and A. Rodríguez-Bernal, "Bifurcation and stability of equilibria with asymptotically linear boundary conditions at infinity", Proc. Roy. Soc. Edinburgh Sect. A 137:2 (2007), 225-252. MR 2009d:35194 Zbl 1202.35119

[Arrieta et al. 2009] J. M. Arrieta, R. Pardo, and A. Rodríguez-Bernal, "Equilibria and global dynamics of a problem with bifurcation from infinity", J. Differential Equations 246:5 (2009), 20552080. MR 2010c:35016 Zbl 1195.35040

[Arrieta et al. 2010] J. M. Arrieta, R. Pardo, and A. Rodríguez-Bernal, "Infinite resonant solutions and turning points in a problem with unbounded bifurcation", Internat. J. Bifur. Chaos Appl. Sci. Engrg. 20:9 (2010), 2885-2896. MR 2011m:35107 Zbl 1202.35085

[Castro and Pardo 2011] A. Castro and R. Pardo, "Infinitely many stability switches in a problem with sublinear oscillatory boundary conditions", preprint MA-UCM 2011-2, Univ. Complutense de Madrid, 2011, available at http://www.ucm.es/centros/cont/descargas/documento23873.pdf.

[Crandall and Rabinowitz 1971] M. G. Crandall and P. H. Rabinowitz, "Bifurcation from simple eigenvalues”, J. Functional Analysis 8 (1971), 321-340. MR 44 \#5836 Zbl 0219.46015

[García-Melián et al. 2009] J. García-Melián, J. D. Rossi, and J. C. Sabina de Lis, "An elliptic system with bifurcation parameters on the boundary conditions", J. Differential Equations 247:3 (2009), 779-810. MR 2010f:35073 Zbl 1171.35037

[Korman 2008] P. Korman, "An oscillatory bifurcation from infinity, and from zero", NoDEA Nonlinear Differential Equations Appl. 15:3 (2008), 335-345. MR 2009h:35137 Zbl 1166.34010

[Rabinowitz 1971] P. H. Rabinowitz, "Some global results for nonlinear eigenvalue problems", $J$. Functional Analysis 7 (1971), 487-513. MR 46 \#745 Zbl 0212.16504

[Rabinowitz 1973] P. H. Rabinowitz, "On bifurcation from infinity", J. Differential Equations 14 (1973), 462-475. MR 48 \#7047 Zbl 0272.35017

Received June 3, 2011. Revised November 22, 2011.

\author{
Alfonso CASTRo \\ DEPARTMENT OF MATHEMATICS \\ HARVEY Mudd COLlEGE \\ 301 Platt Boulevard \\ Claremont, CA 91711 \\ UNITED STATES \\ castro@math.hmc.edu
}

\section{ROSA PARDO}

Departamento de Matemática Aplicada

UNIVERSIDAD COMPLUTENSE DE MADRID

AVENIDA COMPLUTENSE S/N

28040 MADRID

SPAIN

rpardo@mat.ucm.es 


\title{
PACIFIC JOURNAL OF MATHEMATICS
}

\author{
http://pacificmath.org \\ Founded in 1951 by \\ E. F. Beckenbach (1906-1982) and F. Wolf (1904-1989)
}

\section{EDITORS}

V. S. Varadarajan (Managing Editor)

Department of Mathematics

University of California

Los Angeles, CA 90095-1555

pacific@math.ucla.edu

Vyjayanthi Chari

Department of Mathematics

University of California

Riverside, CA 92521-0135

chari@math.ucr.edu

\section{Robert Finn}

Department of Mathematics Stanford University

Stanford, CA 94305-2125

finn@math.stanford.edu

Kefeng Liu

Department of Mathematics

University of California

Los Angeles, CA 90095-1555

liu@math.ucla.edu
Darren Long

Department of Mathematics

University of California

Santa Barbara, CA 93106-3080

long@math.ucsb.edu

Jiang-Hua Lu

Department of Mathematics

The University of Hong Kong

Pokfulam Rd., Hong Kong jhlu@maths.hku.hk

Alexander Merkurjev

Department of Mathematics

University of California

Los Angeles, CA 90095-1555

merkurev@math.ucla.edu
Sorin Popa

Department of Mathematics University of California

Los Angeles, CA 90095-1555 popa@math.ucla.edu

Jie Qing

Department of Mathematics

University of California

Santa Cruz, CA 95064

qing@cats.ucsc.edu

Jonathan Rogawski

Department of Mathematics

University of California

Los Angeles, CA 90095-1555

jonr@math.ucla.edu

\section{PRODUCTION}

pacific@math.berkeley.edu

\section{SUPPORTING INSTITUTIONS}

ACADEMIA SINICA, TAIPEI

CALIFORNIA INST. OF TECHNOLOGY INST. DE MATEMÁTICA PURA E APLICADA KEIO UNIVERSITY

MATH. SCIENCES RESEARCH INSTITUTE NEW MEXICO STATE UNIV.

OREGON STATE UNIV.

\author{
STANFORD UNIVERSITY \\ UNIV. OF BRITISH COLUMBIA \\ UNIV. OF CALIFORNIA, BERKELEY \\ UNIV. OF CALIFORNIA, DAVIS \\ UNIV. OF CALIFORNIA, LOS ANGELES \\ UNIV. OF CALIFORNIA, RIVERSIDE \\ UNIV. OF CALIFORNIA, SAN DIEGO \\ UNIV. OF CALIF., SANTA BARBARA
}

\author{
UNIV. OF CALIF., SANTA CRUZ \\ UNIV. OF MONTANA \\ UNIV. OF OREGON \\ UNIV. OF SOUTHERN CALIFORNIA \\ UNIV. OF UTAH \\ UNIV. OF WASHINGTON \\ WASHINGTON STATE UNIVERSITY
}

These supporting institutions contribute to the cost of publication of this Journal, but they are not owners or publishers and have no responsibility for its contents or policies.

See inside back cover or pacificmath.org for submission instructions.

The subscription price for 2012 is US \$420/year for the electronic version, and \$485/year for print and electronic.

Subscriptions, requests for back issues from the last three years and changes of subscribers address should be sent to Pacific Journal of Mathematics, P.O. Box 4163, Berkeley, CA 94704-0163, U.S.A. Prior back issues are obtainable from Periodicals Service Company, 11 Main Street, Germantown, NY 12526-5635. The Pacific Journal of Mathematics is indexed by Mathematical Reviews, Zentralblatt MATH, PASCAL CNRS Index, Referativnyi Zhurnal, Current Mathematical Publications and the Science Citation Index.

The Pacific Journal of Mathematics (ISSN 0030-8730) at the University of California, c/o Department of Mathematics, 969 Evans Hall, Berkeley, CA 94720-3840, is published monthly except July and August. Periodical rate postage paid at Berkeley, CA 94704, and additional mailing offices. POSTMASTER: send address changes to Pacific Journal of Mathematics, P.O. Box 4163, Berkeley, CA 94704-0163.

PJM peer review and production are managed by EditFLOW ${ }^{\mathrm{TM}}$ from Mathematical Sciences Publishers.

PUBLISHED BY PACIFIC JOURNAL OF MATHEMATICS

at the University of California, Berkeley 94720-3840

A NON-PROFIT CORPORATION

Typeset in LATEX

Copyright $(02012$ by Pacific Journal of Mathematics 


\section{PACIFIC JOURNAL OF MATHEMATICS}

Volume $257 \quad$ No. $1 \quad$ May 2012

Energy and volume of vector fields on spherical domains

FABiano G. B. BRito, ANDRÉ O. Gomes and Giovanni S. Nunes

Maps on 3-manifolds given by surgery

BOLDIZSÁR KALMÁR and ANDRÁS I. STIPSICZ

Strong solutions to the compressible liquid crystal system

Yu-Ming ChU, Xian-Gao LiU and XIAO LIU

Presentations for the higher-dimensional Thompson groups $n V$

Johanna HenNig and FranCESCo MatuCCI

Resonant solutions and turning points in an elliptic problem with oscillatory

boundary conditions

ALFONSO CASTRO and ROSA PARDO

Relative measure homology and continuous bounded cohomology of topological pairs

\section{Roberto Frigerio and CRISTINA PAgLiAnTINi}

Normal enveloping algebras

ALEXANDRE N. GrishKov, Marina RASSKazova and SALVATORE SICILIANO

Bounded and unbounded capillary surfaces in a cusp domain

YASUNORI AOKI and DAVID SIEGEL

On orthogonal polynomials with respect to certain discrete Sobolev inner product

Francisco Marcellán, Ramadan Zejnullahu, Bujar Fejzullahu and EDMUNDO HUERTAS

Green versus Lempert functions: A minimal example

PASCAL THOMAS

Differential Harnack inequalities for nonlinear heat equations with potentials under the Ricci flow

JIA-YONG WU

On overtwisted, right-veering open books

PAOLO LISCA

Weakly Krull domains and the composite numerical semigroup ring $D+E\left[\Gamma^{*}\right]$

JUNG WOOK LIM

Arithmeticity of complex hyperbolic triangle groups 